\title{
Online Astronomy for Formal and Informal Learners
}

\author{
Chris Impey ${ }^{1, *}$, and Matthew Wenger ${ }^{1}$ \\ ${ }^{1}$ Department of Astronomy, University of Arizona, Tucson, AZ 85721, United States
}

\begin{abstract}
An increasing amount of formal and informal education is being delivered online. A majority of college students in the U.S. are now taking one or more courses online or flipped (video lectures online, handson activities or labs in the classroom). Meanwhile, massive open online classes, or MOOCs, are transforming the landscape of informal science learning. In contrast to university classes, MOOCs have low completion rates and involve "free choice" learners who are typically adults with jobs, rather than full time students. Based on several years of experience of teaching astronomy online, lessons have been learned on how to engage students in the asynchronous and "disembodied" environment. For nonscience students taking an introductory astronomy course, flipped models optimize interactions by putting the lectures online and allowing classroom time to be used entirely for labs, discussion, and small group activities. Research has shown that normalized learning gains are greatest in such learner-centered classes. We have enrolled over 110,000 learners from 150 countries in two astronomy MOOCs: a Udemy course called "Astronomy: State of the Art" and a Coursera offering called "Astronomy: Exploring Time and Space." The core content is a set of video lectures, augmented by quizzes, activities, and peer writing assignments. We have a large amount of research data on learner demographics and motivations, and on the types of engagement that correlate with completing the courses. In a peer writing assignment, the learners comment on recent discoveries in astronomy. A rubric and a model answer are provided, and each person grades writing of three other learners. Learners who complete either the first activity or the first peer writing assignment are highly engaged in online discussions and social media, completing the course at a rate ten times higher than average.
\end{abstract}

\section{Science literacy}

An important context for teaching astronomy is the public understanding of science. In the United States, most of the 22 million college students who are not science majors have to take science as part of a breadth or General Education requirement. Astronomy is one of the most popular options for satisfying the requirement [1]. Among members of the public, astronomy is popular due to discoveries and space missions that often feature in the news and due to the outreach efforts of hundreds of planetariums and science centers.

\footnotetext{
* Corresponding author: cimpey@,as.arizona.edu
} 
At the University of Arizona we have been exploring undergradaute student understandings and beliefs about science and technology since 1989, drawing on a database of over 12,000 responses to a science literacy survey that was derived from policy-driven instruments. The instrument contains a general measurement of science knowledge tethered in NSF surveys of the general public [2], in particular an open-ended response to the question "What does it mean to study something scientifically?" The instrument also has a set of statements about science, technology, and society coded using a Likert scale that measures attitudes about scientific issues and levels of belief in pseudoscience (Figure 1).
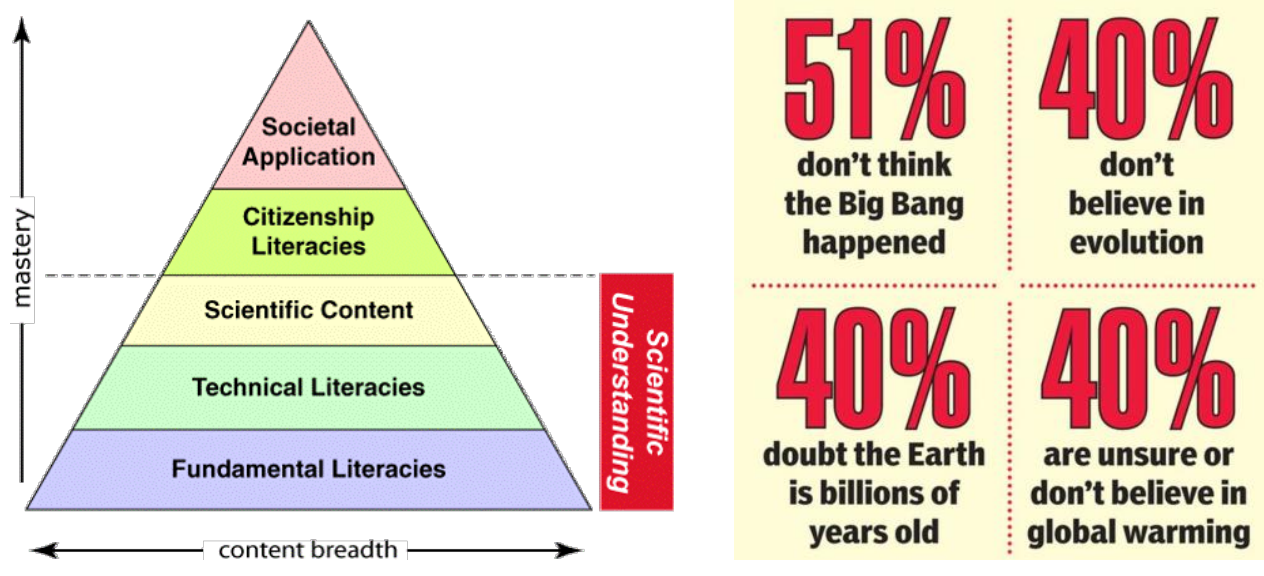

Fig. 1. Public understanding of science is rooted in fundamental literacy about how science works, numeracy, and familiarity with the concepts and facts of modern science. Building on this base, it is possible to make decisions on issues of societal importance (left). In the United States, the general public is sceptical about, or dissents from, evidence-based views held by an overwhelming majority of scientists (right) [3].

Our analysis reveals little change in students' basic science knowledge scores over twentyseven years. While the average level of science knowledge is higher than for the general public measured as by the NSF, there is only a $15 \%$ gain in what students know as they graduate, relative to what they knew when they entered as freshmen. Student demographic variables, including major and the number of science courses completed, predicts $10 \%$ of the variance in students' overall science knowledge scores. Overall, students' belief levels in pseudoscience and supernatural phenomena are high and appear to be resistant to the college instruction that every non-science major experiences. This is perhaps unsurprising given that most students' contact to scientific information is on the Internet and takes place outside of class. Strikingly, non-scientific beliefs correlate poorly with performance on the science knowledge part of the survey. The NSF surveys of the general public have reported data showing a long term increase in science literacy which has been attributed to college science requirements for non-science majors. Our data do not support that conjecture [4-6].

We analyzed students' responses to four open-ended questions and compared their ideas to those of scientists asked the same questions. Non-science majors are able to describe science using many of the words we consider to be important, although not in the same way as scientists. We have also developed and revised a new instrument to find out where students get their science information and how they rate the reliability of those sources. 
Unsurprisingly, we found that most students use sources outside the science classroom, online, for their information about science, especially science knowledge for their own interests. We have found no strong relationships between students' sources of knowledge and their basic science knowledge, but we have found a relationship between students' science knowledge and their information literacy. Specifically, students who lack a critical ability to judge online sources of science have lower science knowledge scores and describe studying something scientifically in a less sophisticated way than their peers. Our research reveals a complex relation between knowledge and belief, leading to recommendations on how best to train students for informed participation in a civic society, where science and technology issues are prominent.

\section{Active learning}

In college classrooms around the United States and Europe, teaching continues to be mostly based on lectures, with occasional breaks for questions or discussion. As a teaching model, it is cheap, easy to implement, and familiar. Yet data convincingly show that active learning methods, where students interact with the instructor and with their peers in various ways, are more effective. Recdent meta-analysis of hundreds of science classes showed that active learning reduces the number of students who fail by a third and increases the number who excel by a factor of two. According to the lead author 'The impact of these data should be like the Surgeon General's report on smoking and health in 1964 - they should put to rest any debate about whether active learning is more effective than lecturing' [7].

\section{Standardized Coefficients}

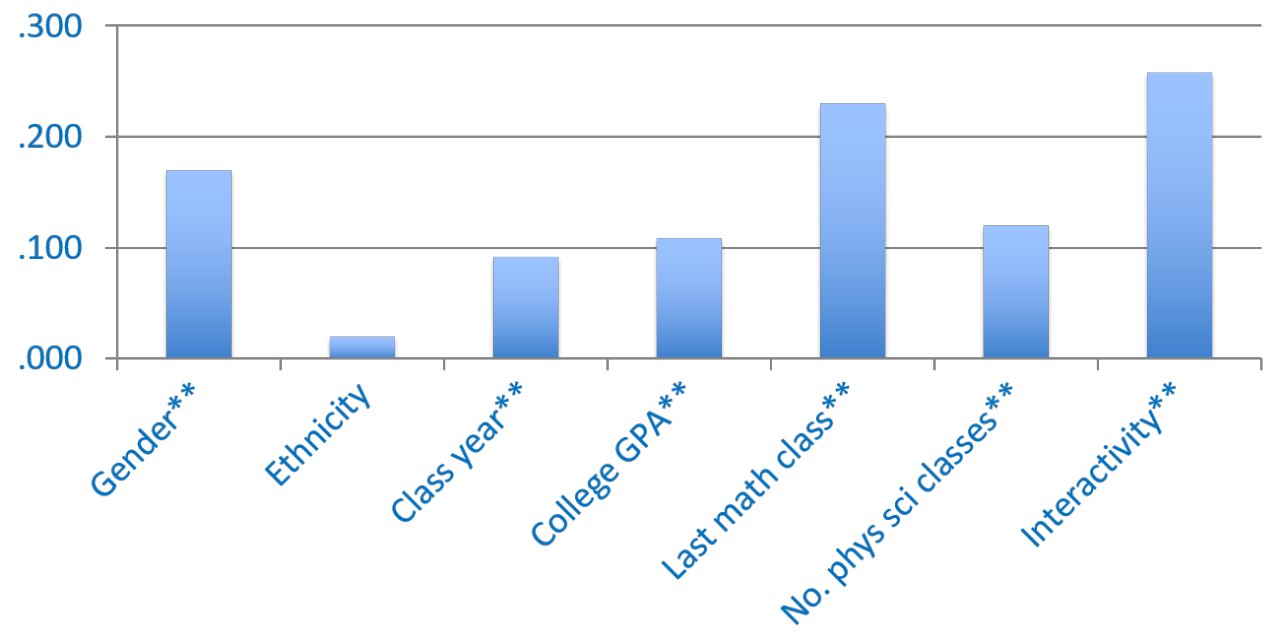

Fig. 2. Normalized learning gains in a set of astronomy classes, based on pre- and post-testing using a standardized concept inventory, in terms of the factors responsible for learning. Those marked with a double asterisk are significant at a level $p<0.01$. Classroom interactivity, using a variety of tools, has the largest impact on the learning gains [8].

Faculty and staff at the Center for Astronomy Education at the University of Arizona have spent years designing and validating evidence-based, active engagement learning strategies for introductory astronomy. These activities include Lecture-Tutorials, Think-Pair-Share tasks, Ranking Tasks, and online-delivered engagement tools like interactive simulations 
and sorting tasks, including those developed for the Mastering Astronomy curriculum and the Nebraska Astronomy Applets Project [9]. The design of these evidence-based activities is guided by empirically supported theories of learning. The intention is to help students identify when their pre-instructional ideas are appropriate, and lead them to correct mental models. It is also to recognize when they are inappropriate and might interfere with the construction of correct mental models. According to sociocultural theories of learning, instructional activities take place in situations in which students must engage in discourse, negotiate shared understandings of conceptually difficult astronomical ideas, and complete tasks collaboratively that they could not otherwise do individually.

\section{Online learning}

The rapidly increasing capability of the Internet and the availability of online content are rapidly eroding the "sense of place" in a college education. Online learning is not new but it has started to become a major component of the educational landscape. The number of students taking one or more online classes in the United States grew from 2 million up to 7 million over the years 2002 to 2011, and over the same period the online percentage of the total enrollment grew from $10 \%$ to $32 \%$. More recently, the falling number of 18 year olds means that overall college enrollment is declining, but in the face of this, online enrollment is still growing (Figure 3). Reticence over the quality of online instruction is diminishing. In one 2012 survey, $77 \%$ of the academic leaders at 2800 U.S. colleges considered purely online education to be the same as, or superior to, face-to-face education [10]. Set against this growth is the fact discussed earlier that research-based and learner-centered teaching methods have not been adopted widely in the science classroom [11].

\section{ENROLLMENT BY TYPE OF COURSE - DEGREE-GRANTING INSTITUTIONS - 2012-2014}

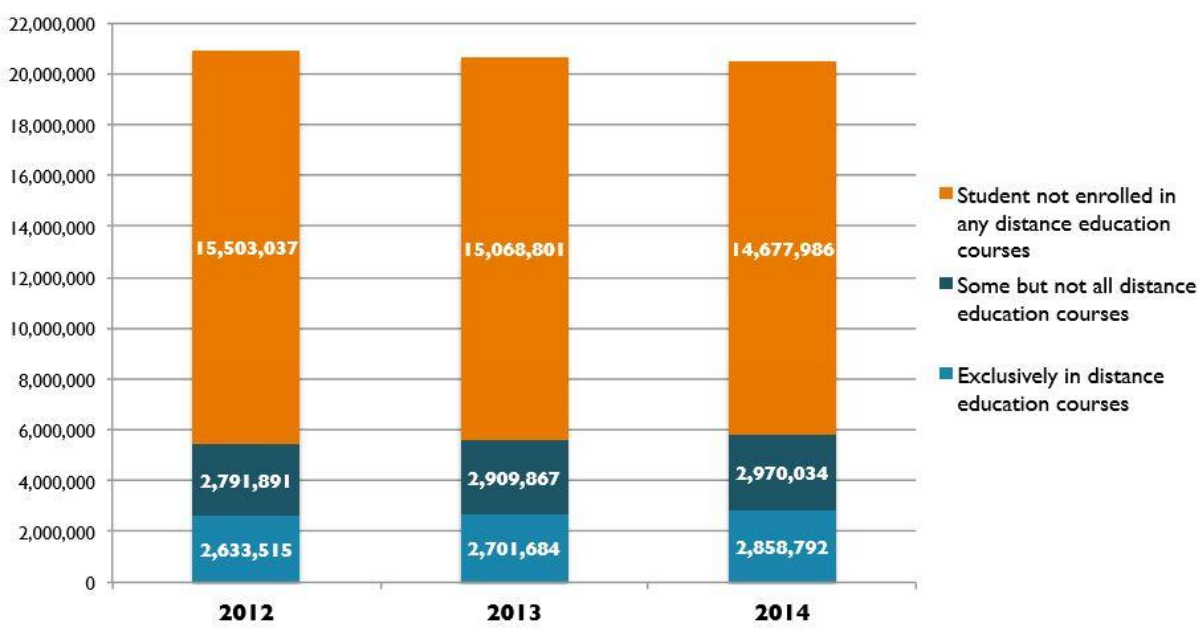

Fig. 3. Demographic forces in the United States mean that college enrollments are falling nationwide, but the number and the fraction of students taking one or more online class, or studying exclusively online, continues to rise [12]. 
The challenge of implementing good pedagogy without face-to-face contact or personalized interaction is one that is unsolved. While the active learning activities described above were originally developed for classes with 200 or fewer students, in the Astronomy Department at the University of Arizona, they have been successfully implemented in mega-courses, in which 700 to 1400 students attend class. A popular middle path for instructors who do not want to lose the direct contact (and greater control) of a face-to-face class is a hybrid class, also called a flipped class. In this model, students access the lectures and course materials online, but they are in a face-to-face classroom two or three times a week for labs, hands-on activities, lecture tutorials, group discussion, and other research-validated methods for enhancing learning. The flipped or hybrid model gives students flexibility, uses the online medium for the content where interaction with the instructor is not required, and optimizes the scarce resource represented by time in a large classroom.

\section{Massive open online classes}

Six years after they burst onto the educational scene, the jury is still out on massive open online classes (MOOCs). The number of MOOCs has grown rapidly to over 7000 (Figure 4), and the number of learners is over 35 million [13]. A MOOC is a university-level course that's offered completely online with no pre-requisites, usually free, though often learners pay for a completion certificate. There are three main categories of providers. For-profit companies like Coursera partner with universities and faculty to offer a wide range of subjects typical of a Liberal Arts curriculum. Non-profit companies like edX operate in a similar way. Meanwhile, the smaller companies like Udemy offer vocational courses and courses addressing professional development, with revenues shared between the company and individual instructors.

\section{Growth of MOOCs}

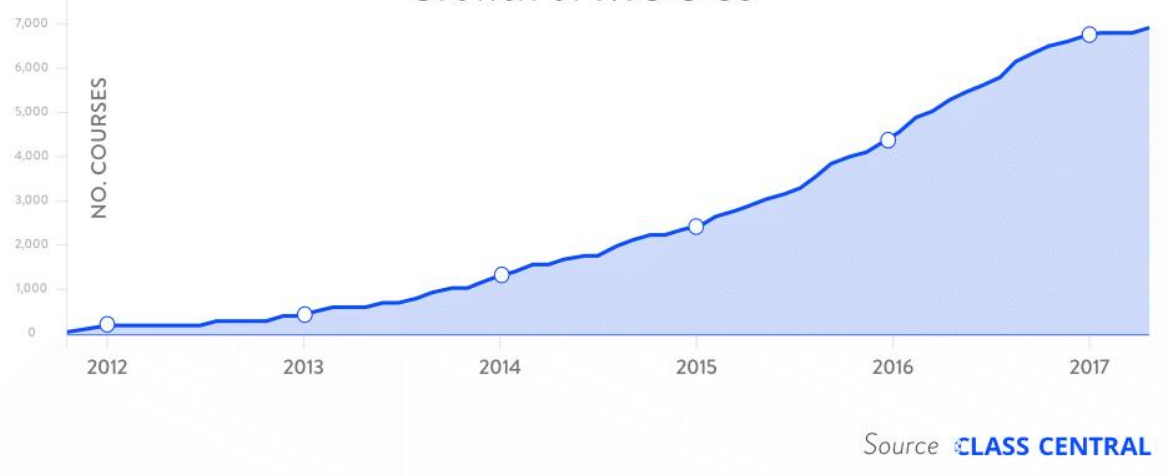

Fig. 4. Rapid growth in the number of MOOCs, or massive open online classes. MOOCs are online courses that are free, or where a small amount is charged for a completion certificate, and where there is no college credit awarded [14].

Debate over the efficacy of MOOCs and their role in the educational landscape continues unabated [15]. The benefit of free exposure to faculty expertise from major universities is mitigated by generally low completion rates of MOOCs, in the range of 5-10\% [16]. However, it is unrealistic to expect very high completion rates in a free-choice learning situation, with adult learners who have to juggle families and jobs and who are not as heavily invested in the educational experience in terms of paying tuition and receiving 
college credit. Many people clearly use the MOOC "marketplace" to browse options or sample an interesting subject without making substantial commitment (Figure 5). Modular MOOCs allow a lot of aggregate consumption of content and learning, even for those who are not planning to complete the course. Moreover, a clear majority of those who do complete MOOCs report educational benefits and most also claim career benefits [17].

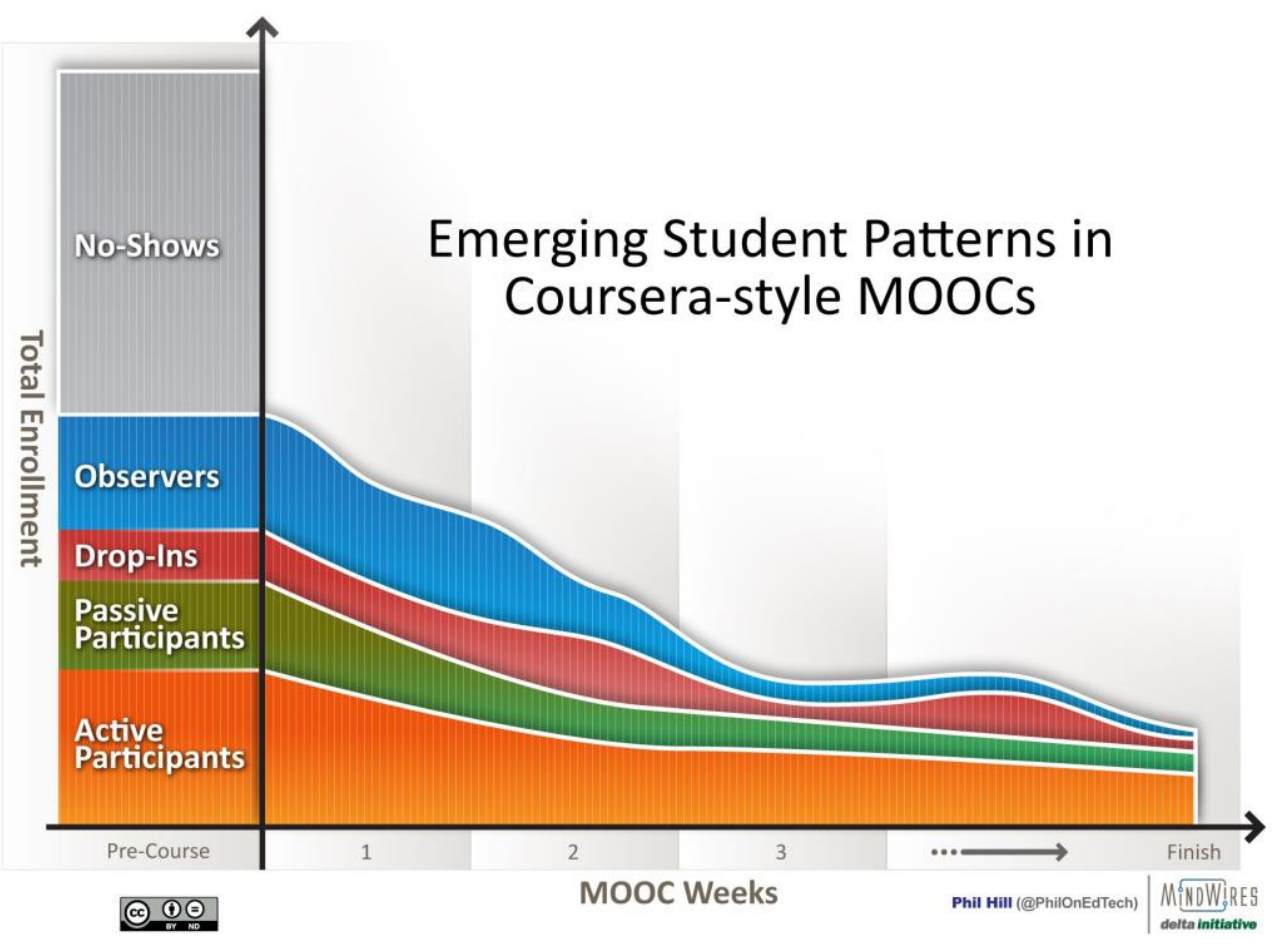

Fig. 5. The completion rates of MOOCs are in the range 5-10\%, far lower than college classes. But a MOOC attracts learners with different motivations and contexts for learning than a university. Many people never open the course materials after enrolling, and others simply watch videos without doing any assignments. Even among active participants, there is a falloff in participation over the duration of the MOOC (courtesy P. Hill).

MOOCs are an important testbed for instructional strategies and pedagogy, Providers like Coursera offer a data-rich environment for researchers; learner privacy can be safeguarded while their behavior can be analyzed at the level of clicks on the web site. One criticism is that MOOCs typically depend on video lectures and multiple choice quizzes, augmented by online discussions. For the most part, the pedagogy is transmissive and the learners are passive, similar to many traditional university learning environments. This ignores research demonstrating that the best learning gains come from methods that actively engage students and let them work with peers and also get direct feedback from instructors. Currently, MOOC platforms do not tend to support interactive modes of instruction or provide easy ways to incorporate external or third party tools. Few would argue that online instruction rivals the best classroom experience, although some experts contend that virtual classes can be better than real ones [18]. On the plus side, new tools and technologies can be tested readily on any MOOC platform, generating copious data and permitting a very rapid development cycle. 
Astronomy is a dynamic subject, with new research results reaching the level of the popular media almost daily. Particularly rapid progress is being made in the areas of exoplanets and cosmology. "Astronomy: Exploring Time and Space" was the first MOOC to be offered by the University of Arizona after it joined the Coursera consortium. The goal is to offer a survey of the subject with an emphasis on topics where advances in knowledge have been particularly rapid or profound. The major modules in the course are: the scientific method and the history of astronomy, the night sky, telescopes and the tools of astronomy, matter and radiation, the Solar System, extrasolar planets, the birth and death of stars, galaxies and large scale structure of the universe, cosmology and the big bang, and life in the universe. Relative to an introductory astronomy textbook, the course gives particular emphasis to the method of science, large new telescopes, exoplanet detection and characterization, tests of cosmological models, and the prospects of life in the Solar System and beyond.

As in most MOOCs, the core content is a series of video lectures. Ten high level modules are divided into 10 to 12 topics each, and the video segments are at the level of a topic, typically 6 to 15 minutes long. Video was shot with HD resolution against a green screen so that backgrounds and graphics could be added later. Images and animations and video clips were incorporated into the videos in the production phase. Video editing was done in Final Cut Pro X. The goal for the video lectures was a natural style and a varied presentation so they would appeal to people accustomed to science shows on television and high quality web videos. The 109 video lectures total just under 18 hours, which is at the high end for most MOOCs. No textbook is used. Instead, the learners have access to a free electronic textbook and other online astronomy resources provided by the instructor (Chris Impey) at the "Teach Astronomy" web site [19].

Evaluation is based on thirteen multiple choice quizzes keyed to the video lectures. Learner engagement is increased with three writing assignments and two online activities. Each writing assignment is peer-reviewed using tools provided by Coursera that randomly assigns three reviewers to each piece of writing. The writing assignments are graded on a five-point scale using a rubric provided by the instructor that each reviewer uses to score the work of their peers. The three topics for the 500-word writing assignments are: the best choice of telescopes for observing in different parts of the electromagnetic spectrum, the detection of exoplanets and their properties, and the exotic end states of stars. In addition, students complete two online activities. One utilizes the citizen science project Galaxy Zoo [20]; the other has the learners using the NASA MicroObservatory robotic telescope network to select a target, use the telescope network to take an image of an object in the sky, then write a brief report on the properties and the significance of the target chosen.

The course uses other modes of engagement which do not affect the final grade or the ability to attain completion certificates. Coursera provides embedded discussion boards, and threads were created for each content module as well as for general course issues. Topics generated by leaners proliferate during the course, such as on practical observing with small telescopes, on UFOs, on science and religion, and on astronomy in the news. We can sort the threads by activity level and popularity, and members of the instructional team try to participate in every discussion thread at least once. Live question and answer sessions are conducted using Google Hangouts every few weeks of the course, and are automatically archived and posted publicly on YouTube. The course has a Facebook page and a Twitter account, and the instructor maintains an active presence on social media, typically making 3-4 posts per week. 


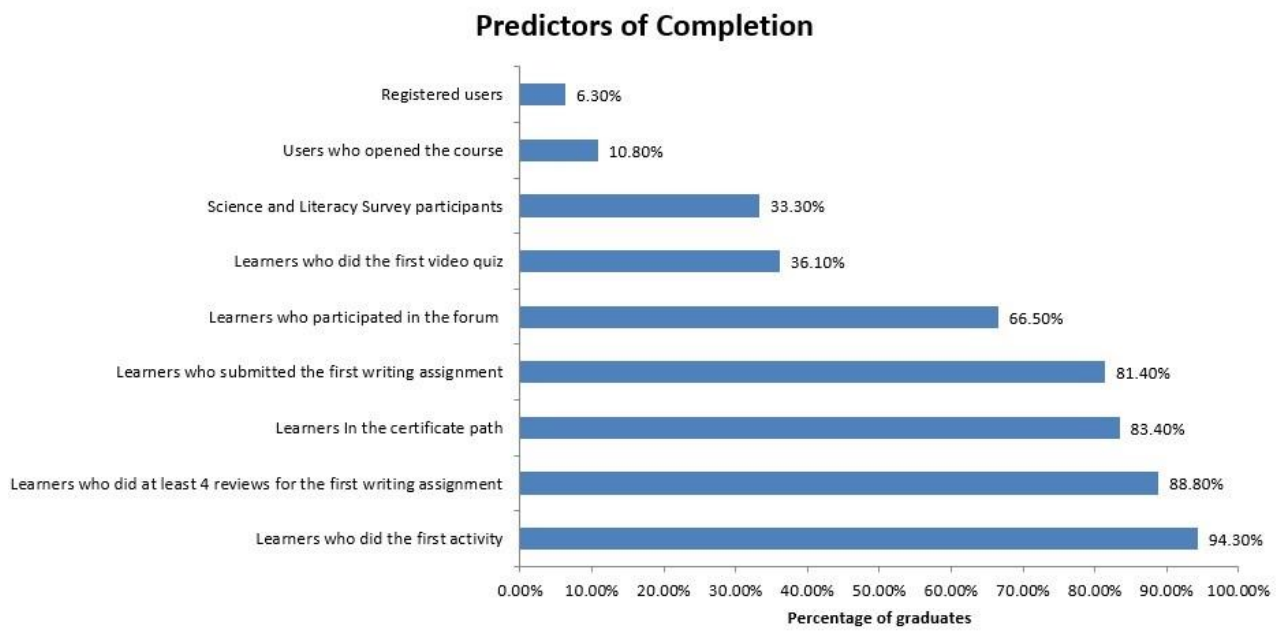

Fig. 6. Predictors of completion for the Coursera MOOC "Astronomy: Exploring Time and Space." The normally low level of completion is much higher for anyone who does an activity or a writing assignment suggesting that early engagement options may boost completion [21].

We looked at predictors of completion, omiting "phantoms" who never opened the course or completed any assignment. That baseline is the $11 \%$ of people who opened the course and then completed the course and graduated. Participation of any kind boosts the odds of completion, but there are different levels of predictors. In the lowest tier, 33\% of those who did the Science Literacy survey at the beginning of the course completed the course and $36 \%$ of the people who did the first video quiz completed the course. In the middle tier, there was a $67 \%$ completion rate for people who participated, even just once, in the discussion forums. In the highest tier as far as percentage who graduated are: $81 \%$ of those who did the first writing assignment, $83 \%$ of those who chose the certificate path, $89 \%$ of those who did required peer reviews for the first writing assignment, and $94 \%$ of those who did the first activity (Figure 6). The message drawn from this data is that early engagement in the more intensive assessments like writing and projects is a strong predictor of success in this MOOC. This lesson can carry over to instructional design in face-to-face classrooms.

\section{A web site for education and outreach}

An important tool for formal and informal teaching of astronomy is a free, open access web site designed for formal and informal learners of astronomy, called Teach Astronomy [22]. The web site features an online textbook of 450,000 words, with 2000 quiz questions and a glossary of 900 terms, ten thousand images, a curated collection of the 42,000 astronomy articles in Wikipedia, a complete video lecture course, a video FAQ tool, and other material provided by content partners. Clustering algorithms and an interactive visual interface allow users to browse closely related items of content. The web site automatically displays appropriately on smart phones and small format devices like tablets.

The most sophisticated technology behind Teach Astronomy is a software mechanism for the indexing, clustering, and graphical delivery of astronomy content. A set of text-based items is sent to an indexing system called Lucene, by the Apache Foundation. Lucene provides powerful algorithms to search through large collections of semantically related text. It calculates a "distance" between any two items in the index based on keyword 
overlap, so it is easy to list the "nearest neighbors" to any item. The output of a search and this clustering analysis is a graphical display called a Wikimap. The central node of the Wikimap is the closest match to a search and the nearest neighbors are shown as radial spokes (the default number is ten, for viewing convenience). Clicking an outlying node centers that item, and a new set of nearest neighbors is displayed. The Wikimap provides an appealing method for browsing or "surfing" related content. This method has been applied to a walled garden consisting of 42,000 curated astronomy articles in Wikipedia (Figure 7).

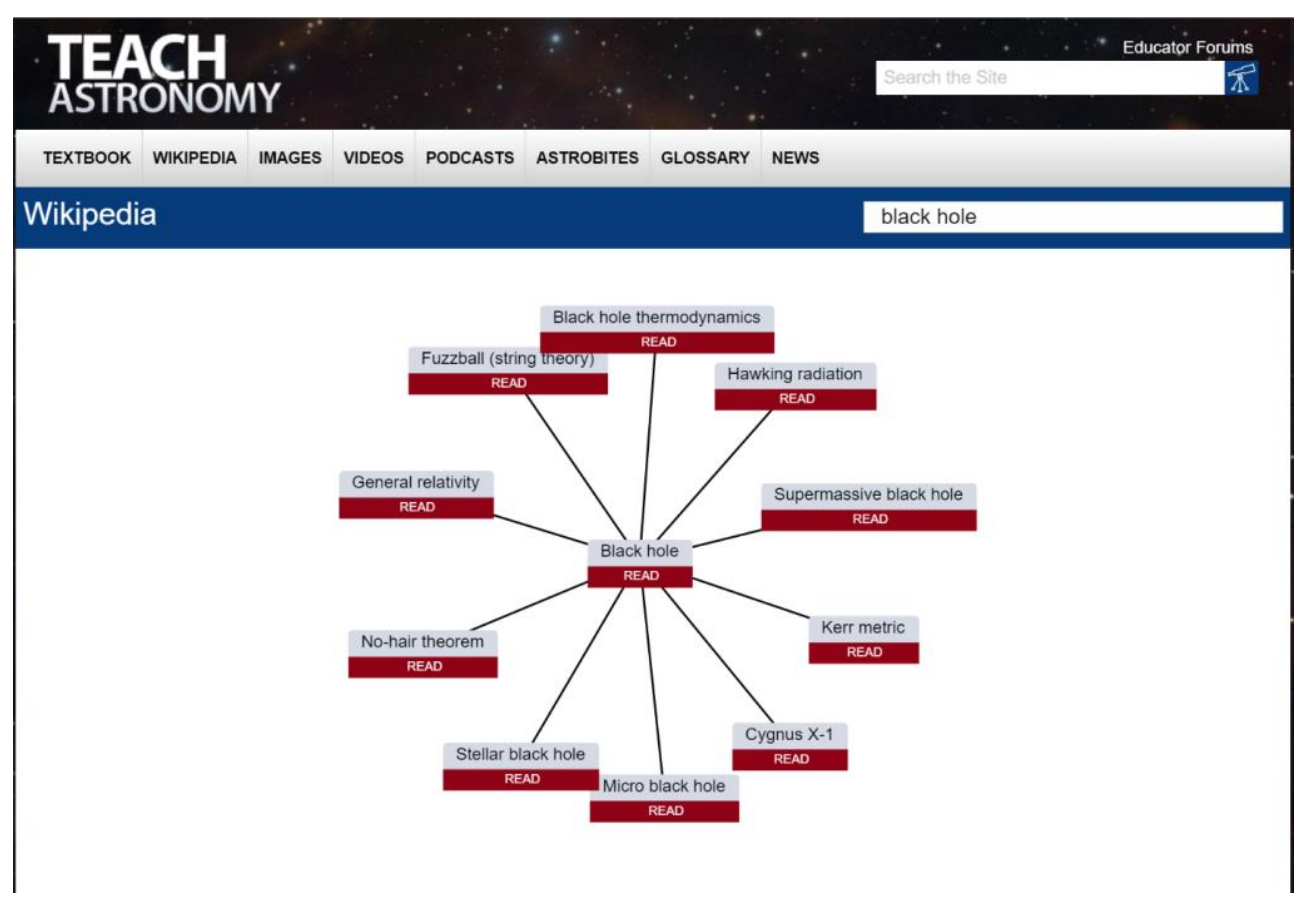

Fig. 7. The Teach Astronomy web site has a clustering tool based on keyword overlap of text-based content. In this example, the astronomy articles in Wikipedia are clustered and a search on black holes returns the black hole article as the central node, with 10 closely related articles as outlying nodes.

This approach is powerful and flexible. A Lucene index and the subsequent clustering can be created for any type of text-based content. The obvious application is for articles about astronomy, but images with keyword-rich captions work equally well, and for videos with transcripts, the text of the transcripts is clustered. The nodes of the Wikimap are active, so clicking on them can pop up an article, an image, a video, or a URL. The Wikimap runs on HTML5, CSS3, and JavaScript. A full physics engine runs alongside the core renderer, dynamically keeping the nodes evenly spaced. The site aims to satisfy the requirements of both formal learners and informal learners such as amateur astronomers [23].

The author acknowledges many conversations with his central partners on all of this work, Matthew Wenger and Sanyln Buxner, along with input, comments, and major contributions from Alexander Danehy and Martin Formanek. This work was supported by grants from the National Science Foundation (Award \#1244799) and the Howard Hughes Medical Institute (Award \#4215580). Infrastructure and staff support was also provided by Steward Observatory and the College of Science at the University of Arizona. 


\section{References}

1. A. Fraknoi, Astronomy Education Review, 1, 121 (2001)

2. National Science Board, Science and Engineering Indicators (National Science Foundation, Washington, D.C., 2016)

3. The AP-GfK Poll, http://www.ap-gfkpoll.com (2014)

4. C. Impey, S. Buxner, J. Antonellis, E. Johnson, and C. King, Journal of College Science Teaching, 40, 70 (2011)

5. C. Impey, Organizations, People, and Strategies in Astronomy, Volume 2, p. 353 (Duttlenheim, Venngeist, 2013)

6. C. Impey, M. Formanek, S. Buxner, and M. Wenger, Electronic Journal of Science Education, 40, 4 (2017)

7. S. Freeman, et al., Proceedings of the National Academy of Sciences, 111, 8410 (2014)

8. E. Prather, A. Rudolph, G. Brissenden, and W. Schlingman, American Journal of Physics, 77, 320 (2009)

9. E. Prather, A. Rudolph, and G. Brissenden, Physics Today, 62, 41 (2009)

10. Grade Change: Tracking Online Education in the United States (Babson Survey Research Group, 2013)

11. Reaching Students: What Research Says About Effective Instruction in Undergraduate Science and Engineering (National Academies Press, Washington, D.C., 2015)

12. Online Report Card: Tracking Online Education in the United States (Babson Survey Research Group, 2016)

13. MOOCs in 2015: Breaking Down the Numbers, http://www.edsurge.com (2015)

14. By the Numbers: MOOCs in 2016, http://www.classcentral.com/report (2016)

15. D. Gammage, S. Fernando, and I. Perera, $8^{\text {th }}$ International Conference on Ubi-Media, 224 (2015)

16. H. Khalil and M. Ebner, Proceedings of World Conference on Educational Multimedia, Hypermedia, and Telecommunications, 1305 (2014)

17. C. Zhenghao et al., Harvard Business Review online, September 22 (2015)

18. B. Oakley, Nautilus online, October 29 (2015)

19. C. Impey, K. Hardegree-Ullman, K. Patikkal, A. Srinithan, C. Austin, N. Ganesan, and B. Guvenen, Astronomy Education Review, 03011 (2013)

20. J. Raddick, et al., Astronomy Education Review, 9, 1 (2009)

21. C. Impey, M. Wenger, M. Formanek, and S. Buxner, Communicating Astronomy to the Public, 21, 20 (2016)

22. Teach Astronomy, http://www.teachastronomy.com

23. C. Impey, K. Hardegree-Ullman, A. Patikkal, and C. Austin, International Journal for Innovation Education and Research, 4, 117 (2016) 